\title{
The Use of Acid Fluorides Increases the Scope of the Reductive Acylation of Esters
}

\author{
Yongda Zhang and Tomislav Rovis* \\ Department of Chemistry, Colorado State University \\ Fort Collins, Colorado 80523
}

\section{Supporting Information}

General Methods. All reactions were carried out under an atmosphere of argon in flame-dried glassware with magnetic stirring. Dichloromethane was degassed with argon and passed through two columns of neutral alumina. Pyridine was dried over solid $\mathrm{KOH}$ and stored over $4 \AA$ molecule sieves. Column chromatography was performed on EM Science silica gel 60 (230-400 mesh). Thin layer chromatography was performed on EM Science $0.25 \mathrm{~mm}$ silica gel $60-\mathrm{F}$ plates. Visualization was accomplished with UV light, $\mathrm{KMnO}_{4}$, aqueous ceric ammonium molybdate, or bromocresol green dips followed by heating.

Benzoyl fluoride was purchased from Aldrich Chemical Co. and used without further purification. Other acyl fluorides were prepared by known literature methods. ${ }^{1}$

Melting points were measured with a MelTemp II melting point apparatus outfitted with a Fluke 51 thermocouple and are uncorrected. Infrared spectra were obtained on a Nicolet Avatar 320 FT-IR spectrometer. ${ }^{1} \mathrm{H}$ NMR and spectra were recorded on a Varian 300, 400, or $500 \mathrm{MHz}$ spectrometer at ambient temperature. Data are reported as follows: chemical shift in parts per million $(\square, \mathrm{ppm})$ from an internal standard [tetramethylsilane (TMS) or deuterated chloroform $\left(\mathrm{CDCl}_{3}\right)$ ], multiplicity ( $\mathrm{s}=$ singlet, $\mathrm{d}=$ doublet, $\mathrm{t}=$ triplet, $\mathrm{q}=$ quartet, and $\mathrm{m}=$ multiplet $)$, integration, and coupling constant $(\mathrm{Hz}) .{ }^{13} \mathrm{C}$ NMR and spectra were recorded on a Varian 300,400 , or $500 \mathrm{MHz}$ spectrometer at ambient temperature. Chemical shifts are reported in ppm from $\mathrm{CDCl}_{3}$ taken as $77.0 \mathrm{ppm}$. Mass spectra were obtained on Fisons VG Autospec.

\section{Standard Procedure for the One-Pot DIBAL-H Reduction and Acylation of an Ester with Acyl Fluoride}

To a solution of starting ester $(1 \mathrm{mmol})$ in $\mathrm{CH}_{2} \mathrm{Cl}_{2}(5 \mathrm{~mL})$ was added DIBAL-H (1.0 $\mathrm{M}$ in hexane, $1.1 \mathrm{~mL}, 1.1 \mathrm{mmol}, 1.1$ equiv) dropwise at $-78{ }^{\circ} \mathrm{C}$ under an argon atmosphere. After the reaction was stirred for $2 \mathrm{~h}$ at $-78{ }^{\circ} \mathrm{C}$, neat pyridine $(0.24 \mathrm{~mL}, 3.0$ mmol, 3.0 equiv) was introduced via syringe followed by slow addition of a solution of DMAP (0.13 g, $1.1 \mathrm{mmol}, 1.1$ equiv) in $1.5 \mathrm{~mL}$ of $\mathrm{CH}_{2} \mathrm{Cl}_{2}$ at $-78{ }^{\circ} \mathrm{C}$. Finally, acyl fluoride ( $1.5 \mathrm{mmol}, 1.5$ equiv) was added dropwise. The resulting mixture was allowed to warm to $15{ }^{\circ} \mathrm{C}$ over $12 \mathrm{~h}$. The reaction was quenched with $5 \mathrm{~mL}$ of saturated $\mathrm{NH}_{4} \mathrm{Cl}$. Although no emulsion formed in the workup, $5 \mathrm{ml}$ of saturated Rochelle's salt solution was added. After the mixture was stirred for $15 \mathrm{~min}$, the organic layer was separated, and

\footnotetext{
${ }^{1}$ Chen, C.; Chien, C.-T.; Su, C.-H. J. Fluorine Chem. 2002, 115, 75.
} 
the aqueous layer was extracted with $\mathrm{CH}_{2} \mathrm{Cl}_{2}(4 \times 5 \mathrm{~mL})$. The combined organic layers were washed with ice-cooled $\mathrm{NaHSO}_{4}$, saturated $\mathrm{NaHCO}_{3}$, brine, and dried over $\mathrm{Na}_{2} \mathrm{SO}_{4}$. After removal of the volatiles, the product was purified by silica gel chromatography.

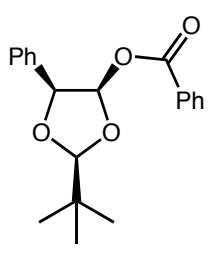

(2S*, 4R*, 5S*)-4-benzoyloxy-2-tert-butyl-5-phenyl-1, 3-dioxolane (3a)

According to standard procedure described above, ester $1(0.22 \mathrm{~g}, 1 \mathrm{mmol})$ was treated with DIBAL-H (1.0 $\mathrm{M}$ in hexane, $1.1 \mathrm{~mL}, 1.1 \mathrm{mmol}, 1.1$ equiv), followed by addition of neat pyridine $(0.24 \mathrm{~mL}, 3.0 \mathrm{mmol}, 3.0$ equiv), DMAP (0.13 g, $1.1 \mathrm{mmol}, 1.1$ equiv), and benzoyl fluoride (0.16

$\mathrm{mL}, 1.5 \mathrm{mmol}, 1.5$ equiv). The product was purified by column chromatography on silica gel (1\% ethyl acetate/hexanes) to afford 3a $(0.30 \mathrm{~g}, 94 \%)$ as a white solid. ${ }^{1} \mathrm{H}$ NMR $\left(300 \mathrm{MHz}, \mathrm{CDCl}_{3}\right) \square 7.77-7.74(\mathrm{~m}, 2 \mathrm{H}), 7.50-7.46(\mathrm{~m}, 3 \mathrm{H}), 7.35-7.25(\mathrm{~m}, 5 \mathrm{H}), 6.63(\mathrm{~d}$, $1 \mathrm{H}, J=3.6 \mathrm{~Hz}), 5.16(\mathrm{~d}, 1 \mathrm{H}, J=3.9 \mathrm{~Hz}), 4.98(\mathrm{~s}, 1 \mathrm{H}), 1.07(\mathrm{~s}, 9 \mathrm{H}) ;{ }^{13} \mathrm{C} \mathrm{NMR}(75 \mathrm{MHz}$, $\left.\mathrm{CDCl}_{3}\right) \square 165.3,133.8,133.2,129.8,129.7,128.5,128.4,128.2,127.3,111.8,94.1,82.9$, 34.7, 24.7; IR ( $\mathrm{NaCl}$, neat) 1727, 1266, 1024, 970, $710 \mathrm{~cm}^{-1}$; HRMS $\left[\mathrm{C}_{20} \mathrm{H}_{22} \mathrm{O}_{4}-\mathrm{H}\right]^{+}$Calc. 325.1440. Found (EI+) 325.1429.

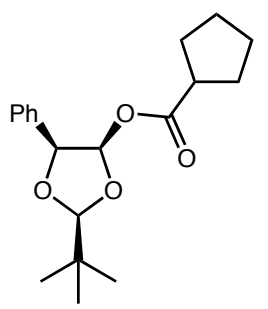

\section{$\left(2 S^{*}, 4 R^{*}, 5 S^{*}\right)-4-c y c l o p e n t y l c a r b o n y l o x y-2-t e r t-b u t y l-5-p h e n y l-1$,} 3-dioxolane (3b)

According to standard procedure described above, ester $1(0.22 \mathrm{~g}, 1$ mmol) was treated with DIBAL-H (1.0 M in hexane, $1.1 \mathrm{~mL}, 1.1 \mathrm{mmol}$, 1.1 equiv), followed by addition of neat pyridine $(0.24 \mathrm{~mL}, 3.0 \mathrm{mmol}$, 3.0 equiv), DMAP (0.13 g, $1.1 \mathrm{mmol}, 1.1$ equiv), and cyclopentanecarbonyl fluoride $(0.17 \mathrm{~g}, 1.5 \mathrm{mmol}, 1.5 \mathrm{guiv})$. The product was purified by column chromatography on silica gel (1\% ethyl acetate/hexanes) to afford 3b $(0.31 \mathrm{~g}, 97 \%){ }^{1} \mathrm{H} \mathrm{NMR}\left(300 \mathrm{MHz}, \mathrm{CDCl}_{3}\right) \square 7.41-7.27(\mathrm{~m}, 5 \mathrm{H}), 6.45(\mathrm{~d}, 1 \mathrm{H}$, $J=3.6 \mathrm{~Hz}), 5.04(\mathrm{~d}, 1 \mathrm{H}, J=3.6 \mathrm{~Hz}), 4.88(\mathrm{~s}, 1 \mathrm{H}), 2.43(\mathrm{~m}, 1 \mathrm{H}), 1.51-1.36(\mathrm{~m}, 8 \mathrm{H}), 1.38$ $(\mathrm{s}, 9 \mathrm{H}) ;{ }^{13} \mathrm{C} \mathrm{NMR}\left(75 \mathrm{MHz}, \mathrm{CDCl}_{3}\right) \square 175.1,133.8,128.2,128.0,127.1,111.3,93.1$, 82.5, 44.0, 34.5, 29.6, 25.8, 24.6; IR (NaCl, neat) 2960, 1739,1125, 1025, 972, $697 \mathrm{~cm}^{-1}$; HRMS $\left[\mathrm{C}_{19} \mathrm{H}_{26} \mathrm{O}_{4}\right]^{+}$Calc. 318.1831. Found (EI+) 318.1825.

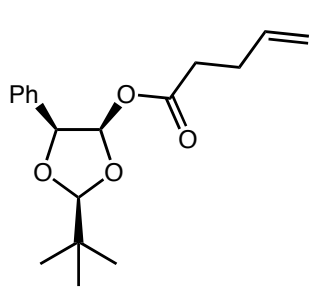

$\left(2 S^{*}, 4 \mathrm{R} *, 5 \mathrm{~S} *\right)-4-(4-p e n t e n o y l o x y)-2-t e r t-b u t y l-5-p h e n y l-1,3-$ dioxolane (3c)

According to standard procedure described above, ester $1(0.22 \mathrm{~g}, 1$ mmol) was treated with DIBAL-H (1.0 M in hexane, $1.1 \mathrm{~mL}, 1.1$ mmol, 1.1 equiv), followed by addition of neat pyridine $(0.24 \mathrm{~mL}$, $3.0 \mathrm{mmol}, 3.0$ equiv), DMAP ( $0.13 \mathrm{~g}, 1.1 \mathrm{mmol}, 1.1$ equiv), and 4pentenoyl fluoride $(0.15 \mathrm{~g}, 1.5 \mathrm{mmol}, 1.5$ equiv). The product was purified by column chromatography on silica gel (1\% ethyl acetate/hexanes) to afford $3 \mathbf{c}(0.28 \mathrm{~g}, 92 \%){ }^{1} \mathrm{H}$ NMR (300 MHz, $\left.\mathrm{CDCl}_{3}\right) \square 7.42-7.26(\mathrm{~m}, 5 \mathrm{H}), 6.45(\mathrm{~d}, 1 \mathrm{H}, J=3.9 \mathrm{~Hz}), 5.55(\mathrm{~m}, 1 \mathrm{H})$, $5.03(\mathrm{~d}, 1 \mathrm{H}, J=4.2 \mathrm{~Hz}), 4.88(\mathrm{~s}, 1 \mathrm{H}), 4.88-4.81(\mathrm{~m}, 2 \mathrm{H}), 2.23-2.00(\mathrm{~m}, 4 \mathrm{H}), 1.06(\mathrm{~s}, 9 \mathrm{H})$; ${ }^{13} \mathrm{C}$ NMR $\left(75 \mathrm{MHz}, \mathrm{CDCl}_{3}\right) \square 171.5,136.4,133.7,128.4,128.1,127.3,115.4,111.4$, 93.4, 82.6, 34.5, 33.7, 28.4, 24.6; IR (NaCl, neat) 2960, 1746, 1129, 1026, 972, $698 \mathrm{~cm}^{-1}$; HRMS $\left[\mathrm{C}_{18} \mathrm{H}_{24} \mathrm{O}_{4}\right]^{+}$Calc. 304.1675. Found (EI+) 304.1668. 


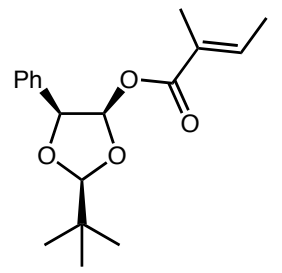

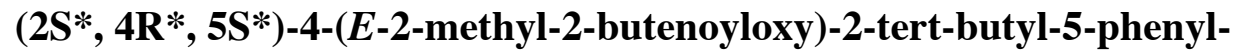
1, 3-dioxolane (3d)

According to standard procedure described above, ester $1(0.22 \mathrm{~g}, 1$ mmol) was treated with DIBAL-H (1.0 M in hexane, $1.1 \mathrm{~mL}, 1.1 \mathrm{mmol}$, 1.1 equiv), followed by addition of neat pyridine $(0.24 \mathrm{~mL}, 3.0 \mathrm{mmol}$, 3.0 equiv), DMAP (0.13 g, $1.1 \mathrm{mmol}, 1.1$ equiv), and tiglyl fluoride ( 0.14 $\mathrm{g}, 1.5 \mathrm{mmol}, 1.5$ equiv). The product was purified by column chromatography on silica gel (1\% ethyl acetate/hexanes) to afford 3d (0.26 g, $87 \%)$. ${ }^{1} \mathrm{H}$ NMR $\left(300 \mathrm{MHz}, \mathrm{CDCl}_{3}\right)$ $\square 7.43-7.26$ (m, 5H), 6.59 (qq, 1H, $J=6.9,1.2 \mathrm{~Hz}), 6.45(\mathrm{~d}, 1 \mathrm{H}, J=3.9 \mathrm{~Hz}), 5.07$ (d, 1H, $J=3.6 \mathrm{~Hz}), 4.92(\mathrm{~s}, 1 \mathrm{H}), 1.66(\mathrm{~d}, 3 \mathrm{H}, J=6.3 \mathrm{~Hz}), 1.59(\mathrm{~m}, 3 \mathrm{H}) ;{ }^{13} \mathrm{C}$ NMR $(75 \mathrm{MHz}$, $\left.\mathrm{CDCl}_{3}\right) \square 166.6,138.2,133.9,128.3,128.1,128.0,127.3,111.5,93.6,82.7,34.6,24.7$, 14.6, 11.9; IR ( NaCl, neat) 2960, 1717, 1252, 1120, 1025, 972, 724, $697 \mathrm{~cm}^{-1}$; HRMS $\left[\mathrm{C}_{18} \mathrm{H}_{23} \mathrm{O}_{4}-\mathrm{H}\right]^{+}$Calc. 303.1596. Found (EI+) 303.1595.

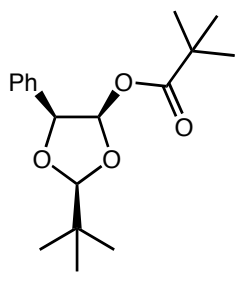

$\left(2 \mathrm{~S} *, 4 \mathrm{R}^{*}, 5 \mathrm{~S} *\right)-4-t e r t-b u t y l c a r b o n y l o x y-2-t e r t-b u t y l-5-p h e n y l-1,3-$ dioxolane (3e)

According to standard procedure described above, ester $1(0.22 \mathrm{~g}, 1$ mmol) was treated with DIBAL-H (1.0 M in hexane, $1.1 \mathrm{~mL}, 1.1 \mathrm{mmol}$, 1.1 equiv), followed by addition of neat pyridine $(0.24 \mathrm{~mL}, 3.0 \mathrm{mmol}$, 3.0 equiv), DMAP (0.13 g, $1.1 \mathrm{mmol}, 1.1$ equiv), and pivaloyl fluoride ( $0.15 \mathrm{~g}, 1.5 \mathrm{mmol}, 1.5$ equiv). The product was purified by column chromatography on silica gel (1\% ethyl acetate/hexanes) to afford 3e $(0.28 \mathrm{~g}, 92 \%)$. ${ }^{1} \mathrm{H}$ NMR $(300 \mathrm{MHz}$, $\left.\mathrm{CDCl}_{3}\right) \square 7.40-7.26(\mathrm{~m}, 5 \mathrm{H}), 6.44(\mathrm{~d}, 1 \mathrm{H}, J=3.9 \mathrm{~Hz}), 5.07(\mathrm{~d}, 1 \mathrm{H}, J=3.9 \mathrm{~Hz}), 4.90(\mathrm{~s}$, $1 \mathrm{H}), 1.06(\mathrm{~s}, 9 \mathrm{H}), 0.86(\mathrm{~s}, 9 \mathrm{H}) ;{ }^{13} \mathrm{C}$ NMR $\left(75 \mathrm{MHz}, \mathrm{CDCl}_{3}\right) \square 177.1,133.9,128.2,128.1$, 126.9, 111.5, 93.2, 82.5, 33.8, 34.5, 26.8, 24.7; IR ( $\mathrm{NaCl}$, neat) 2975, 1736, 1123, 972 , $697 \mathrm{~cm}^{-1}$; HRMS $\left[\mathrm{C}_{18} \mathrm{H}_{26} \mathrm{O}_{4}-\mathrm{H}\right]^{+}$Calc. 305.1753. Found (EI+) 305.1750

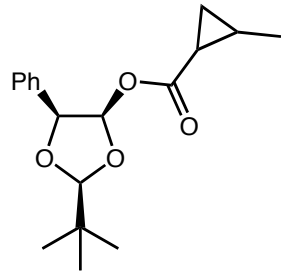

(2S*, 4R*, 5S*)-4-(2-methylcyclopropane)carbonyloxy-2-tertbutyl-5-phenyl-1, 3-dioxolane (3f)

According to standard procedure described above, ester $1(0.22 \mathrm{~g}, 1$ mmol) was treated with DIBAL-H (1.0 M in hexane, $1.1 \mathrm{~mL}, 1.1$ mmol, 1.1 equiv), followed by addition of neat pyridine $(0.24 \mathrm{~mL}, 3.0$ mmol, 3.0 equiv), DMAP (0.13 g, $1.1 \mathrm{mmol}, 1.1$ equiv), and 2methylcyclopropanecarbonyl fluoride $(0.15 \mathrm{~g}, 1.5 \mathrm{mmol}, 1.5 \mathrm{equiv})$. The product was purified by column chromatography on silica gel (1\% ethyl acetate/hexanes) to afford $\mathbf{3 f}$ $(0.20 \mathrm{~g}, 67 \%)$. The product was isolated as a mixture of diastereomers since the dioxolanone and acid fluoride used were racemates.

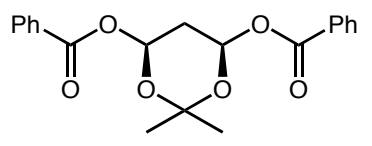

cis-4, 6-benzoyloxy-2, 2-dimethyl-1, 3-dioxane (5)

According to standard procedure described above, ester $4(0.22 \mathrm{~g}$, $1 \mathrm{mmol})$ was treated with DIBAL-H (1.0 M in hexane, $2.2 \mathrm{~mL}, 2.2$ mmol, 2.2 equiv), followed by addition of neat pyridine $(0.48 \mathrm{~mL}, 6.0 \mathrm{mmol}, 6.0$ equiv), DMAP (0.26 g, $2.2 \mathrm{mmol}, 2.2$ equiv), and benzoyl fluoride (0.32 mL, $3.0 \mathrm{mmol}, 3.0$ equiv). The product was purified by column chromatography on silica gel (1\% ethyl 
acetate/hexanes) to afford $5(0.33 \mathrm{~g}, 93 \%)$. ${ }^{1} \mathrm{H}$ NMR $\left(300 \mathrm{MHz}, \mathrm{CDCl}_{3}\right) \square 8.10-8.07$ (m, $4 \mathrm{H}), 7.62-7.56(\mathrm{~m}, 2 \mathrm{H}), 7.44-7.39(\mathrm{~m}, 4 \mathrm{H}), 6.57(\mathrm{t}, 2 \mathrm{H}, J=4.2 \mathrm{~Hz}), 2.54(1 \mathrm{H}, \mathrm{ddd}, J=$ 14.1, 4.2, 4.2 Hz), 2.23 (1H, ddd, $J=14.4,4.8,4.8 \mathrm{~Hz}), 1.73(\mathrm{~s}, 3 \mathrm{H}), 1.58(\mathrm{~s}, 3 \mathrm{H}) ;{ }^{13} \mathrm{C}$ NMR (75 MHz, $\left.\mathrm{CDCl}_{3}\right) \square 165.1,133.5,130.3,130.0,129.7,128.6,99.9,89.1,32.0,29.4$, 27.6; IR ( NaCl, neat) 1728, 1267, 1024, 985, $709 \mathrm{~cm}^{-1}$.

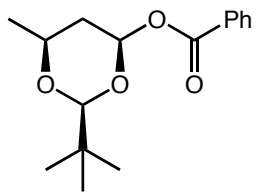

$\left(2 S^{*}, 4 R^{*}, 6 S^{*}\right)-4-b e n z o y l o x y-6-m e t h y l-2-t e r t-b u t y l-1,3-d i o x a n e$ (7)

According to standard procedure described above, ester $6(0.22 \mathrm{~g}, 1$ mmol) was treated with DIBAL-H (1.0 M in hexane, $1.1 \mathrm{~mL}, 1.1 \mathrm{mmol}$, 1.1 equiv), followed by addition of neat pyridine $(0.24 \mathrm{~mL}, 3.0 \mathrm{mmol}, 3.0$ equiv), DMAP ( $0.13 \mathrm{~g}, 1.1 \mathrm{mmol}, 1.1 \mathrm{equiv})$, and benzoyl fluoride $(0.16 \mathrm{~mL}, 1.5 \mathrm{mmol}, 1.5$ equiv). The product was purified by column chromatography on silica gel (1\% ethyl acetate/hexanes) to afforded 7 (0.26 g, $94 \%)$. ${ }^{1} \mathrm{H}$ NMR $\left(300 \mathrm{MHz} \mathrm{CDCl}_{3}\right) \square 8.11-8.08(\mathrm{~m}, 2 \mathrm{H}), 7.58(\mathrm{~m}$, $1 \mathrm{H}), 7.48-7.43(\mathrm{~m}, 2 \mathrm{H}), 6.08(\mathrm{dd}, 1 \mathrm{H}, J=9.9,2.4 \mathrm{~Hz}), 4.31(\mathrm{~s}, 1 \mathrm{H}), 3.83$ (qdd, $J=2.7$, $6.3,12.6 \mathrm{~Hz}), 1.87(\mathrm{ddd}, 1 \mathrm{H}, J=12.6,2.4,2.4 \mathrm{~Hz}), 1.60(\mathrm{~m}, 1 \mathrm{H}), 1.28(\mathrm{~d}, 3 \mathrm{H}, J=6.3$ $\mathrm{Hz}), 0.94$ (s, 9H); ${ }^{13} \mathrm{C}$ NMR (75 MHz, $\left.\mathrm{CDCl}_{3}\right) \square 164.9,133.4,130.1,129.8,128.5,105.3$, 94.0, 71.2, 37.8, 35.0, 25.0, 21.7; IR (NaCl, neat) 2976, 1731, 1272, 1108, 989, $715 \mathrm{~cm}^{-1}$; HRMS $\left[\mathrm{C}_{16} \mathrm{H}_{22} \mathrm{O}_{4}-\mathrm{H}\right]^{+}$Calc. 277.1440. Found (EI+) 277.1444.

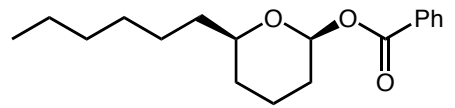

cis-2-benzoyloxy-6-hexanylpyran (9) ${ }^{1}$

According to standard procedure described above, lactone $\mathbf{8}$ $(0.22 \mathrm{~g}, 1 \mathrm{mmol})$ was treated with DIBAL-H $(1.0 \mathrm{M}$ in hexane, $1.1 \mathrm{~mL}, 1.1 \mathrm{mmol}, 1.1$ equiv), followed by addition of neat pyridine $(0.24 \mathrm{~mL}$, 3.0 mmol, 3.0 equiv), DMAP (0.13 g, $1.1 \mathrm{mmol}, 1.1$ equiv), and benzoyl fluoride (0.16 $\mathrm{mL}, 1.5 \mathrm{mmol}, 1.5$ equiv). The product was purified by column chromatography on silica gel ( $1 \%$ ethyl acetate/hexanes) to afford $9(0.24 \mathrm{~g}, 83 \%)$.

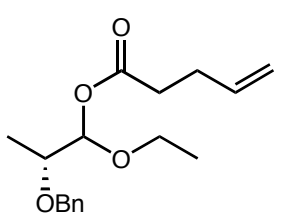

(2R)-1-ethoxy-2-benzyloxypropyl 4-pentenoate (11)

To a solution of ester $\mathbf{1 0}(100 \mathrm{mg}, 0.48 \mathrm{mmol})$ in $\mathrm{CH}_{2} \mathrm{Cl}_{2}(4 \mathrm{~mL})$ at $-78{ }^{\circ} \mathrm{C}$ was added DIBAL-H (1M in hexanes, $\left.1.44 \mathrm{~mL}, 1.44 \mathrm{mmol}\right)$ dropwise via syringe. After $45 \mathrm{~min}$, pyridine (175 $\square 1,2.16 \mathrm{mmol})$ and DMAP (176 mg, 1.44 mmol, 3.0 equiv) in $\mathrm{CH}_{2} \mathrm{Cl}_{2}$ were introduced to the solution dropwise followed by slow addition of 4-pentenoyl fluoride ( 9.0 equiv) via syringe. After the resultant mixture was stirred at $-78^{\circ} \mathrm{C}$ for $14 \mathrm{~h}$, the acetone-dry ice bath was replaced with an ice-water bath. The mixture was stirred at $0{ }^{\circ} \mathrm{C}$ for an additional 30 min, before the reaction was quenched with aqueous $\mathrm{NH}_{4} \mathrm{Cl}(7.5 \mathrm{~mL})$ and saturated Rochelle's salt solution $(6 \mathrm{~mL})$. After the mixture was stirred for $15 \mathrm{~min}$, the organic layer was separated, and the aqueous layer was extracted with $\mathrm{CH}_{2} \mathrm{Cl}_{2}(4 \times 5 \mathrm{~mL})$. The combined organic layers were washed with ice-cooled $\mathrm{NaHSO}_{4}$, saturated $\mathrm{NaHCO}_{3}$, brine, and dried over $\mathrm{Na}_{2} \mathrm{SO}_{4}$. After removal of the volatiles, the product was purified by silica gel chromatography to afford $11(0.12 \mathrm{~g}, 86 \%) .{ }^{1} \mathrm{H} \mathrm{NMR}\left(300 \mathrm{MHz}, \mathrm{CDCl}_{3}\right) \square$ 7.36-7.25 (m, 5H), $2.92(\mathrm{~d}, 1 \mathrm{H}, J=3.9 \mathrm{~Hz}$, minor isomer), $5.83(\mathrm{~d}, 1 \mathrm{H}, J=5.1 \mathrm{~Hz}$, major isomer), $5.80(\mathrm{~m}, 1 \mathrm{H}), 5.12-4.97(\mathrm{~m}, 2 \mathrm{H}), 4.70-4.57(\mathrm{~m}, 2 \mathrm{H}), 3.81-3.70(\mathrm{~m}, 1 \mathrm{H}), 3.67-$

\footnotetext{
${ }^{1}$ Dixon, D. J.; Ley, S. V.; Tate, E. W. J. Chem. Soc., Perkin Trans. 1, 2000, 2385.
} 
$3.53(\mathrm{~m}, 2 \mathrm{H}), 2.51-2.31(\mathrm{~m}, 4 \mathrm{H}), 1.24-1.18(\mathrm{~m}, 6 \mathrm{H}) ;{ }^{13} \mathrm{C} \mathrm{NMR}\left(75 \mathrm{MHz}, \mathrm{CDCl}_{3}\right) \square 172.9$, 138.6, 136.6, 128.4, 127.8, 127.7, 115.7, 98.5, 97.7, 75.4, 75.2, 72.0, 71.9, 65.9, 34.0, 29.0, 15.3, 15.1; IR ( $\mathrm{NaCl}$, teat) 2960, 1733, 1260, 1104, 800, $701 \mathrm{~cm}^{-1}$; HRMS $\left[\mathrm{C}_{17} \mathrm{H}_{24} \mathrm{O}_{4}-\mathrm{H}\right]^{+}$Calc 291.1596. Found (FAB+) 291.1603.

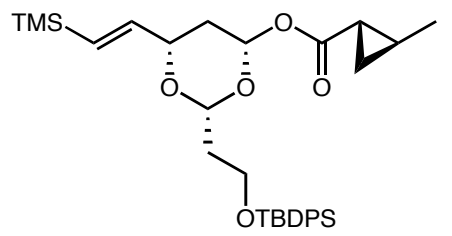

\section{$(2 \mathrm{R}, 4 \mathrm{~S}, 6 \mathrm{~S})-4-[(2 \mathrm{~S}, 1 \mathrm{~S})-2-\mathrm{methyl}-$ cyclopropanecarbonyloxy]-2-[2-(tert-butyldiphenyl- silanyloxy)-ethyl]-6-[2-(trimethylsilanylvinyl]-(1, 3- dioxane (16)}

A flame-dried round bottom flask was charged with acid $12(0.1 \mathrm{~g}, 0.53 \mathrm{mmol})$ and HMDS $(0.16 \mathrm{~mL}, 0.8 \mathrm{mmol})$ in $0.3 \mathrm{~mL}$ of $\mathrm{CH}_{2} \mathrm{Cl}_{2}$, and the solution allowed to stir at room temperature for $13 \mathrm{~h}$. Upon completion, all volatiles including excess HMDS were removed under vacuum. The residue was redissolved in 1 $\mathrm{mL}$ of $\mathrm{CH}_{2} \mathrm{Cl}_{2}$ and cooled down to $-78{ }^{\circ} \mathrm{C}$. The aldehyde $(0.18 \mathrm{~g}, 0.58 \mathrm{mmol})$ was introduced followed by addition of TMSOTf ( $3 \square 1,0.03$ equiv). The resultant mixture was stirred at $-78^{\circ} \mathrm{C}$ for $19 \mathrm{~h}$ and then the reaction was quenched with pyridine $(5 \square 1,0.3$ equiv). The mixture was allowed to warm up to room temperature. All volatiles were removed under vacuum. The residue was passed through a short silica gel column using 4:1 hexane-ethyl acetate as eluent. The residue on removal of solvents was treated with DIBAL-H at $-78{ }^{\circ} \mathrm{C}$ for $2 \mathrm{~h}$. Then, neat pyridine $(0.12 \mathrm{~mL}, 1.5 \mathrm{mmol}, 3.0$ equiv) was introduced via syringe followed by slow addition of a solution of DMAP $(0.07 \mathrm{~g}, 0.58$ mmol, 1.1 equiv) in $0.5 \mathrm{~mL}$ of $\mathrm{CH}_{2} \mathrm{Cl}_{2}$ at $-78{ }^{\circ} \mathrm{C}$. Finally, 2-methyl cyclopropanecarbonyl fluoride $(0.1 \mathrm{~g}, 0.8 \mathrm{mmol}, 1.5$ equiv) was added dropwise, and the resulting mixture was allowed to warm up to $10{ }^{\circ} \mathrm{C}$ over $12 \mathrm{~h}$. The reaction was quenched with $3 \mathrm{~mL}$ of saturated $\mathrm{NH}_{4} \mathrm{Cl}$. Although no emulsion formed in the workup, $3 \mathrm{ml}$ of saturated Rochelle's salt solution was added. After the mixture was stirred for $15 \mathrm{~min}$, the organic layer was separated. The aqueous layer was extracted with $\mathrm{CH}_{2} \mathrm{Cl}_{2}(4 \times 5 \mathrm{~mL})$. The combined organic layers were washed with $\mathrm{NaHSO}_{4}$, saturated $\mathrm{NaHCO}_{3}$, brine, and dried over $\mathrm{Na}_{2} \mathrm{SO}_{4}$. After removal of the volatiles, the product was purified by silica gel chromatography to afford $16(0.15 \mathrm{~g}, 50 \%) .{ }^{1} \mathrm{H} \mathrm{NMR}\left(300 \mathrm{MHz}, \mathrm{CDCl}_{3}\right) \square 7.68-7.66(\mathrm{~m}$, $4 \mathrm{H}), 7.43-7.35(\mathrm{~m}, 6 \mathrm{H}), 6.08-5.91(\mathrm{~m}, 3 \mathrm{H}), 5.03(\mathrm{t}, 1 \mathrm{H}, J=5.4 \mathrm{~Hz}), 4.20(\mathrm{~m}, 1 \mathrm{H}), 2.84(\mathrm{t}$, $2 \mathrm{H}, J=5.4 \mathrm{~Hz}), 2.07-2.00(\mathrm{~m}, 2 \mathrm{H}), 1.91(\mathrm{~m}, 1 \mathrm{H}), 1.65(\mathrm{~m}, 1 \mathrm{H}), 1.41(\mathrm{~m}, 2 \mathrm{H}), 1.25(\mathrm{~m}$, $1 \mathrm{H}), 1.13(\mathrm{~d}, 3 \mathrm{H}, J=6.0 \mathrm{~Hz}), 1.06(\mathrm{~s}, 9 \mathrm{H}), 0.76(\mathrm{~m}, 1 \mathrm{H}), 0.10(\mathrm{~s}, 9 \mathrm{H}) ;{ }^{13} \mathrm{C} \mathrm{NMR}(75$ $\mathrm{MHz}_{\mathrm{CDCl}}$ ) $\square$ 172.6, 143.8, 135.6, 133.8, 133.8, 131.3, 129.6, 127.7, 97.6, 93.2, 77.6, 59.4, 37.3, 36.0, 27.1, 21.5, 19.5, 18.4, 18.0, 17.8, -1.1; IR ( NaCl, neat) 2979, 2932, 1739, 1120, 914, 737, $698 \mathrm{~cm}^{-1}$; HRMS $\left[\mathrm{C}_{32} \mathrm{H}_{46} \mathrm{O}_{5} \mathrm{Si}_{2}\right]^{+}$Calc 566.2884. Found (EI) 566.2869 . 


\section{NMR Spectra}
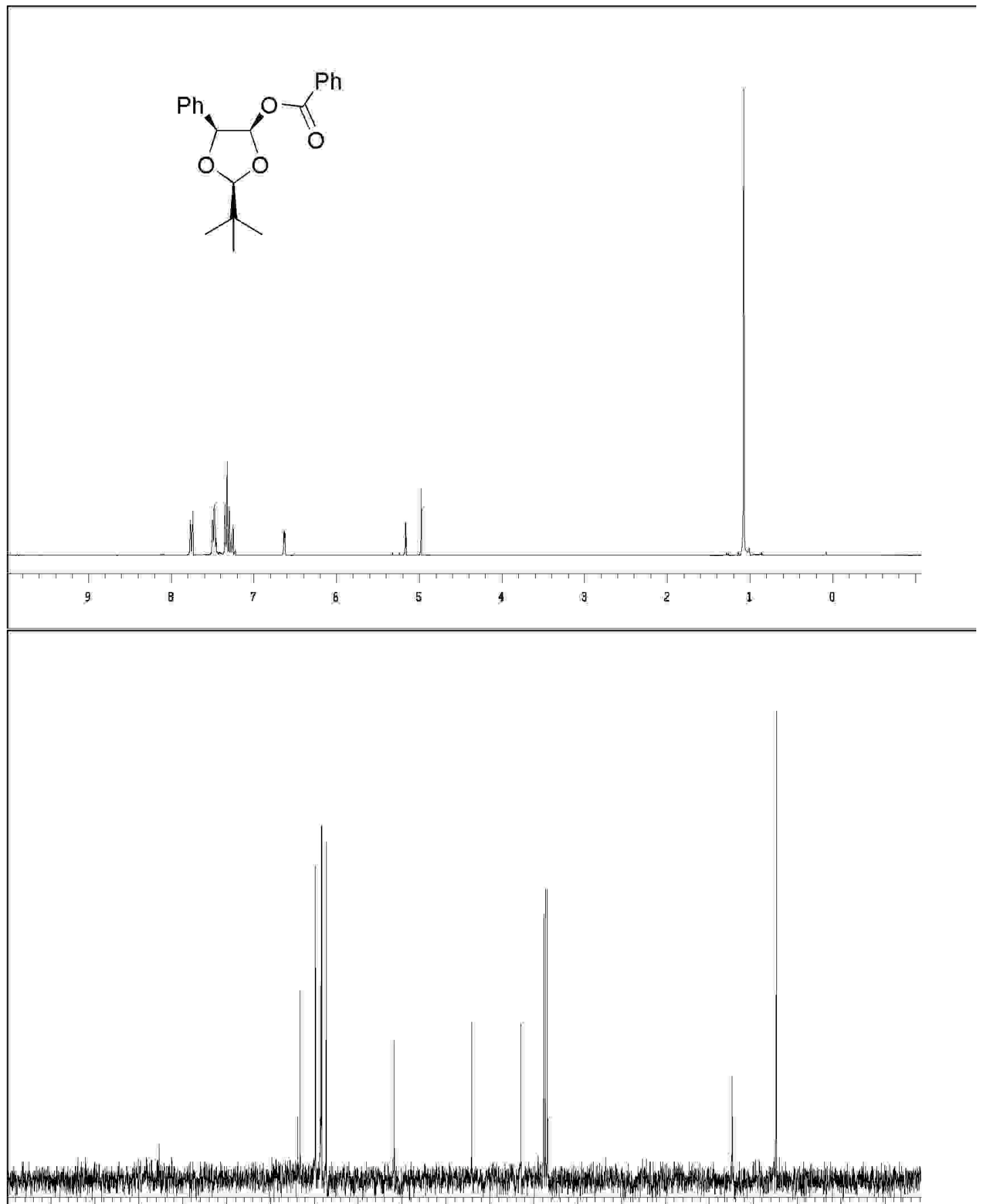


\section{${ }^{1} \mathrm{H}$ NMR and ${ }^{13} \mathrm{C}$ NMR of $\mathbf{3 b}$}
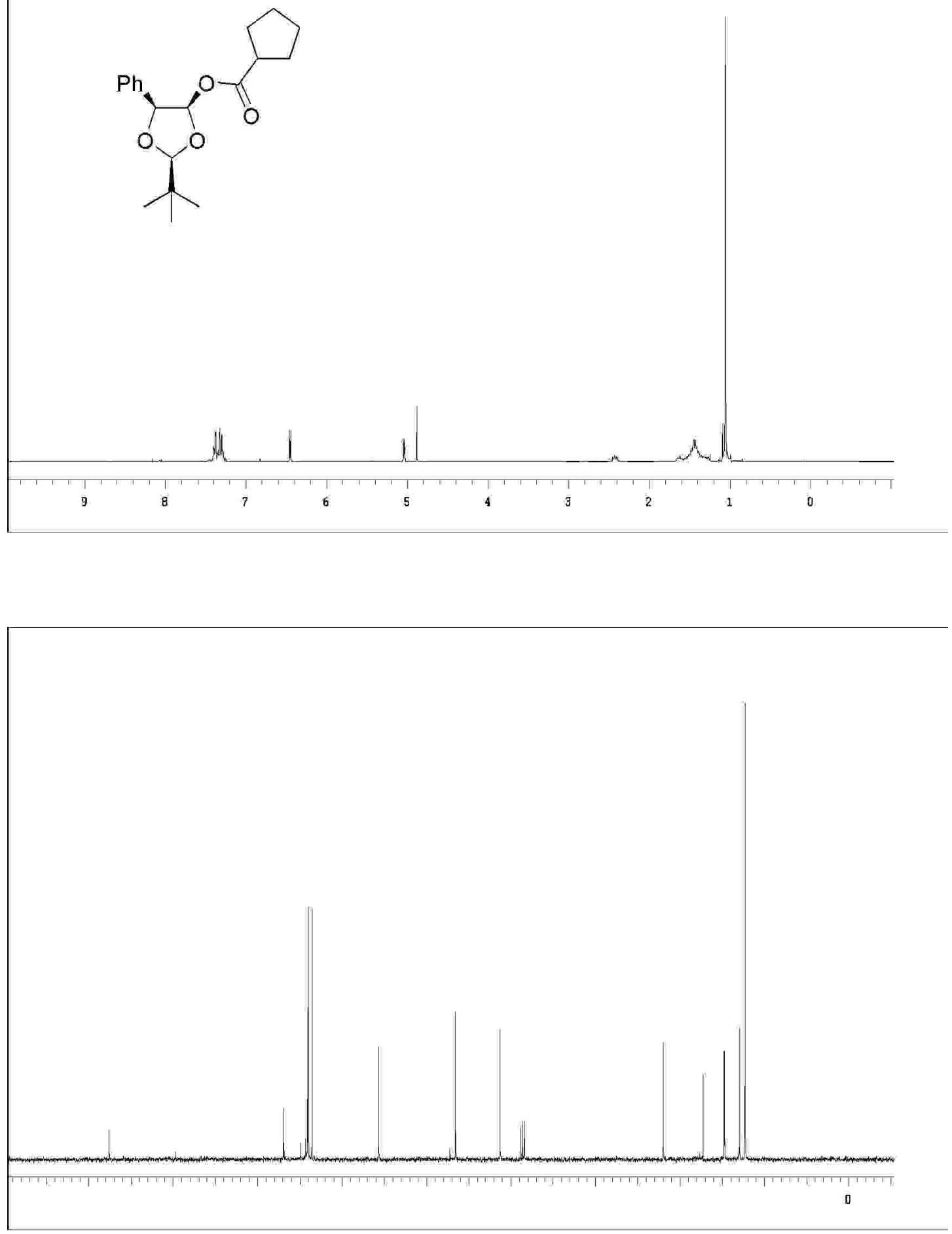
${ }^{1} \mathrm{H}$ NMR and ${ }^{13} \mathrm{C}$ NMR of $\mathbf{3 c}$
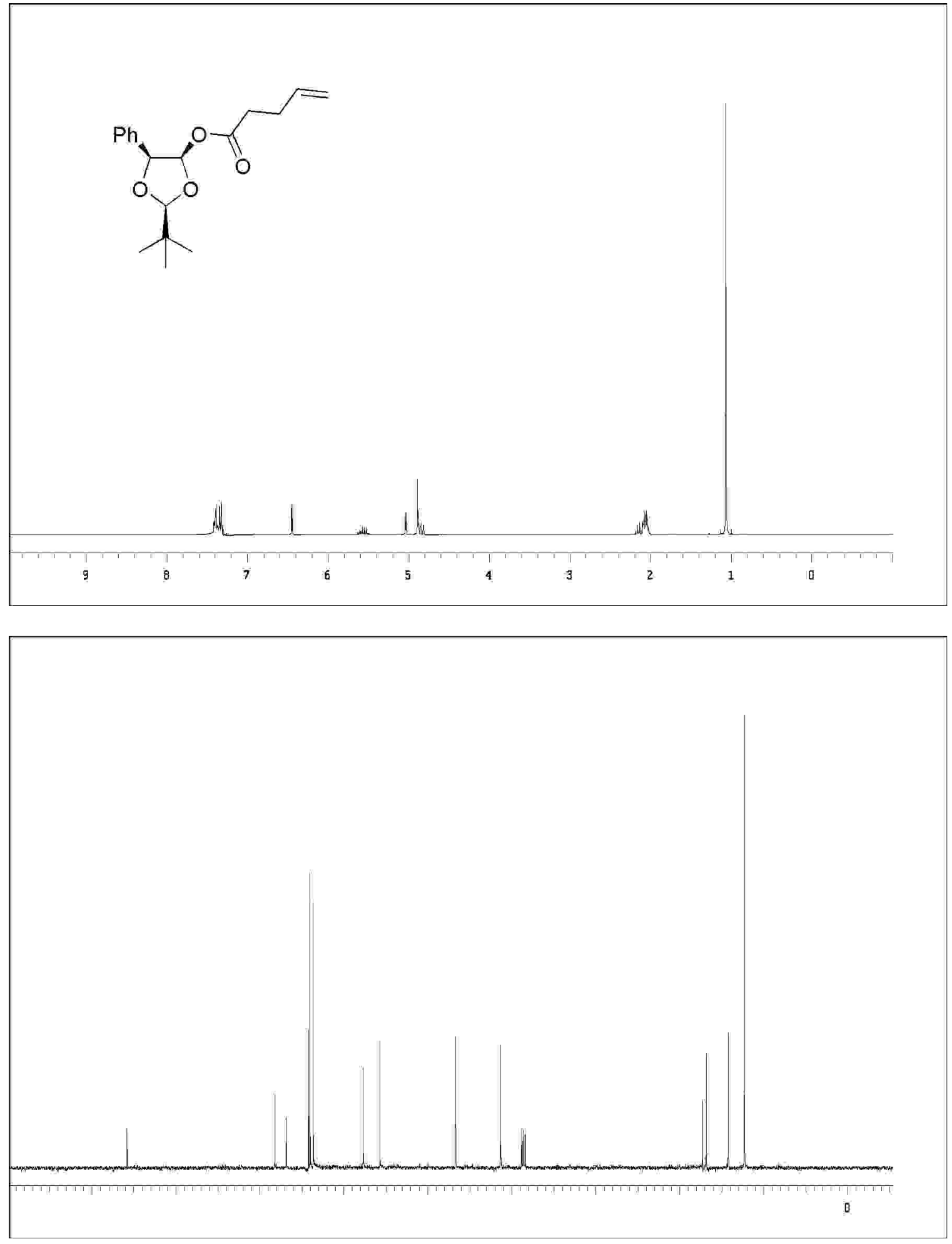


\section{${ }^{1} \mathrm{H}$ NMR and ${ }^{13} \mathrm{C}$ NMR of $\mathbf{3 d}$}
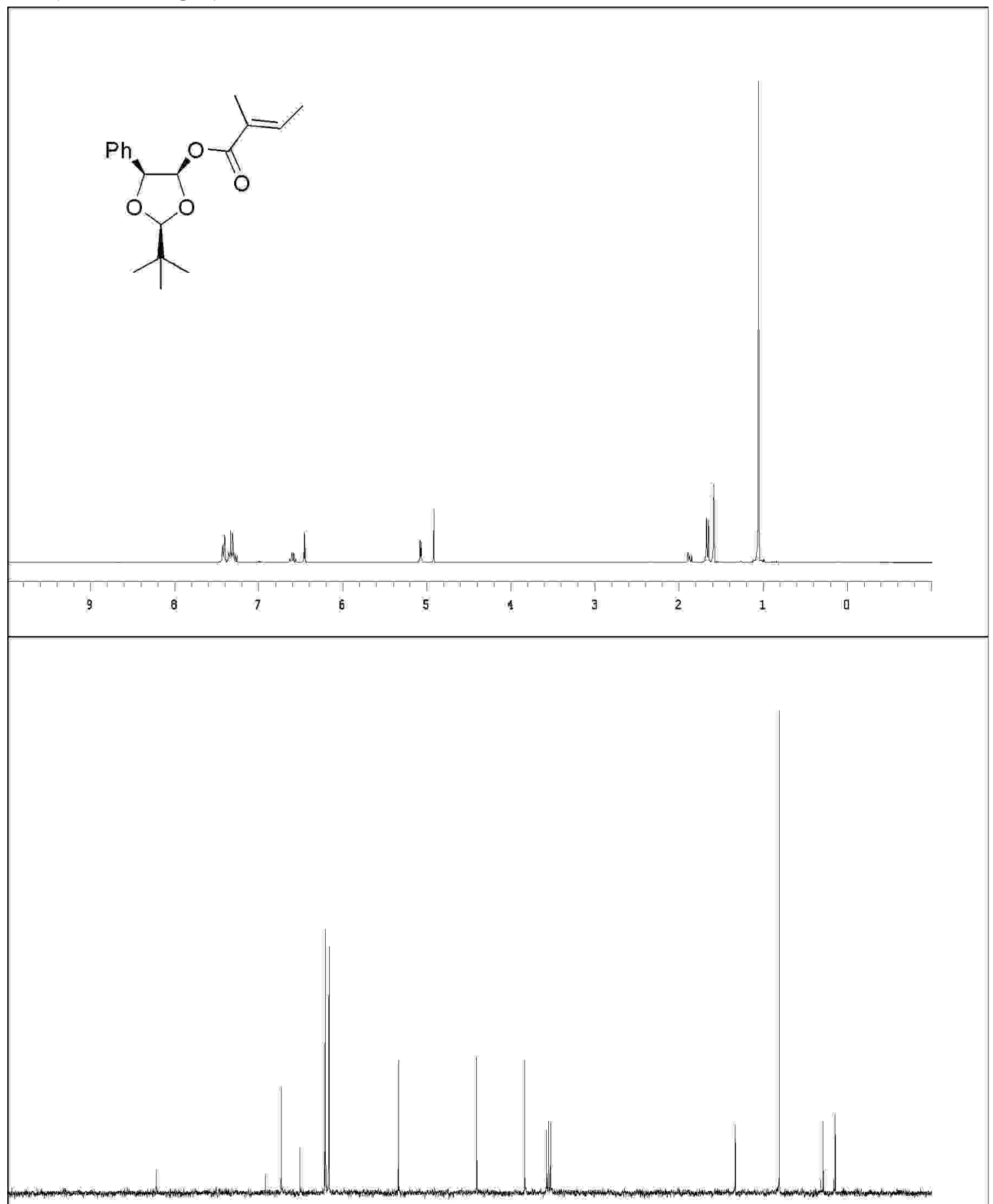
${ }^{1} \mathrm{H}$ NMR and ${ }^{13} \mathrm{C}$ NMR of $\mathbf{3 e}$
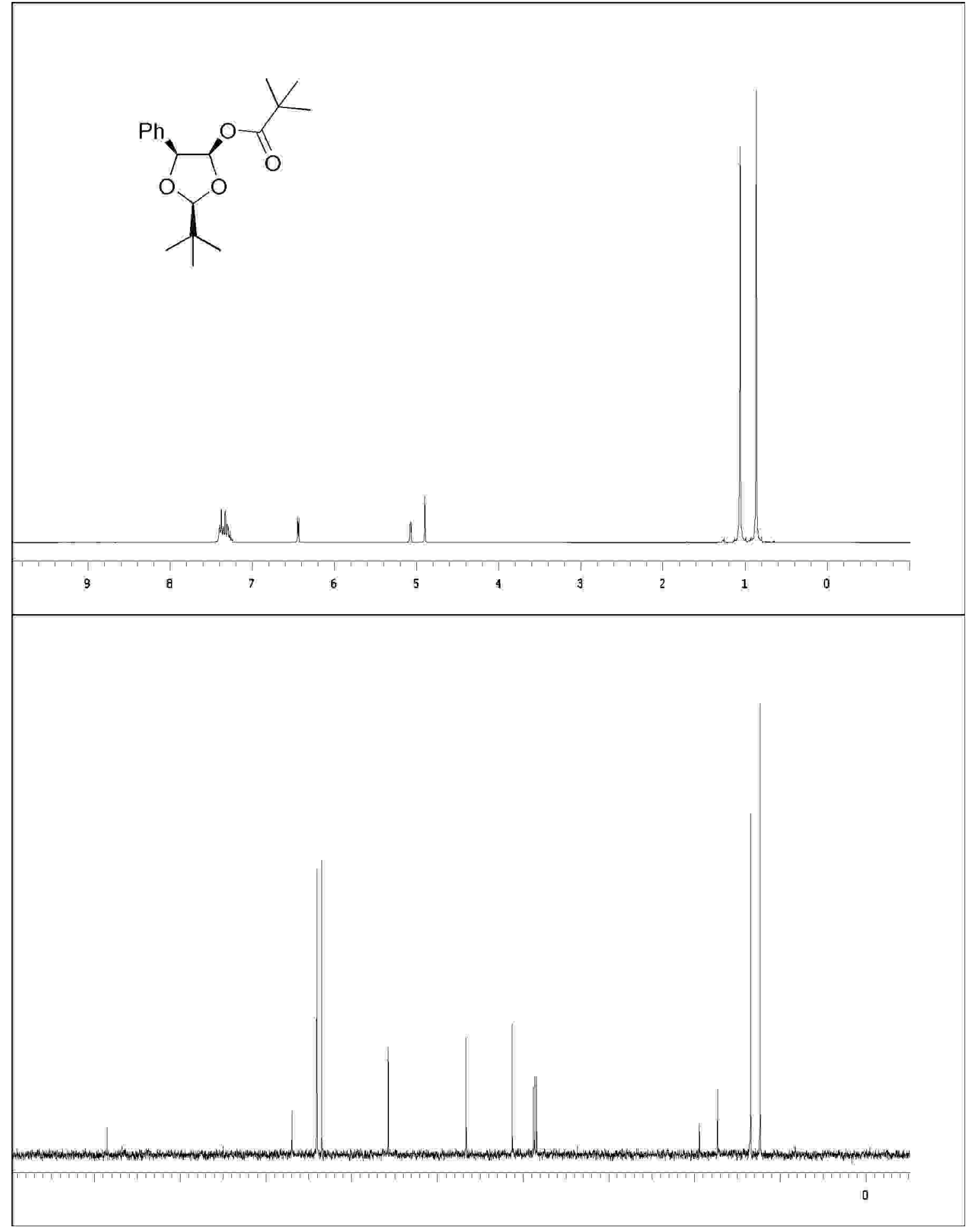
${ }^{1} \mathrm{H}$ NMR and ${ }^{13} \mathrm{C}$ NMR of $\mathbf{3 f}$
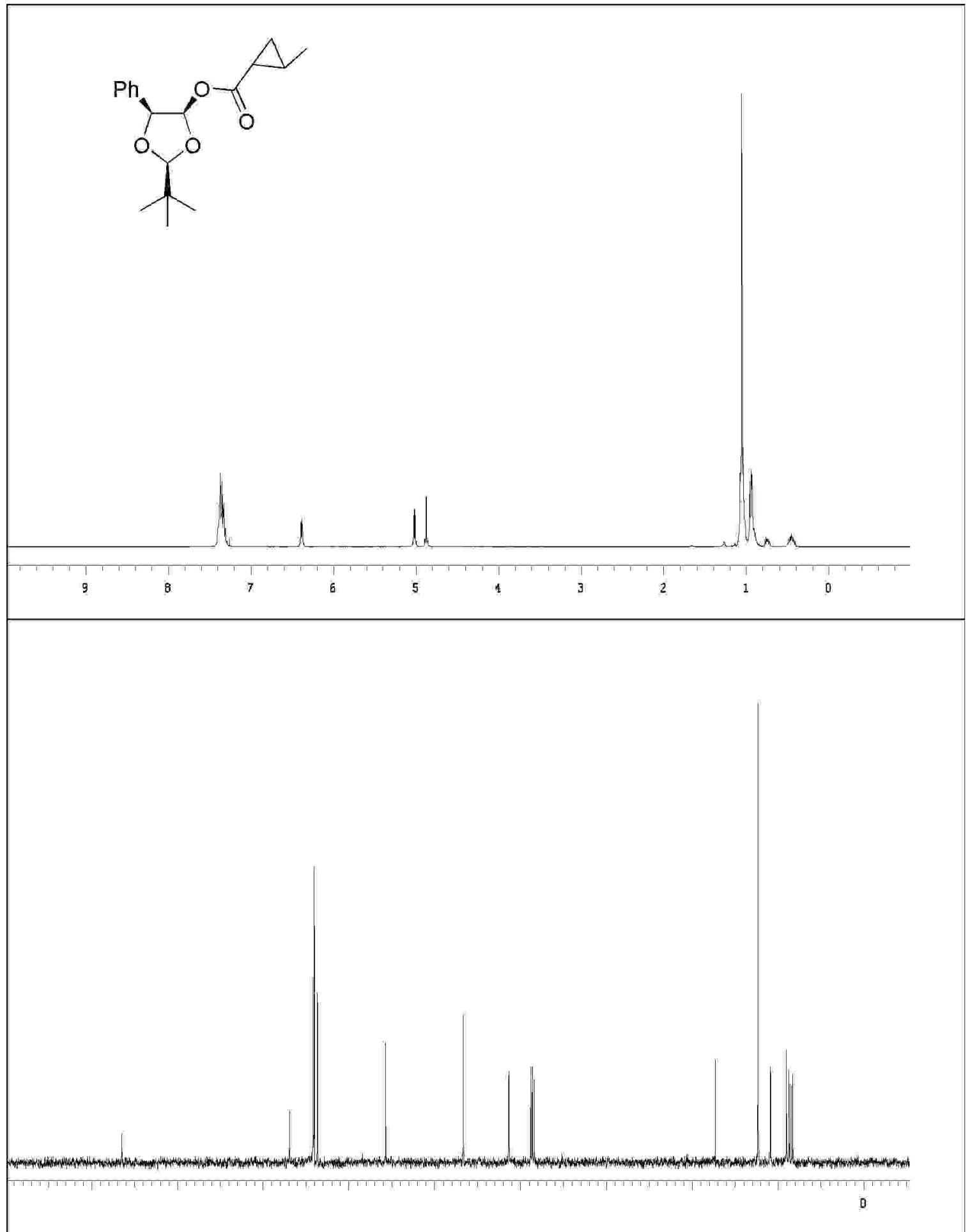
${ }^{1} \mathrm{H}$ NMR and ${ }^{13} \mathrm{C}$ NMR of 5
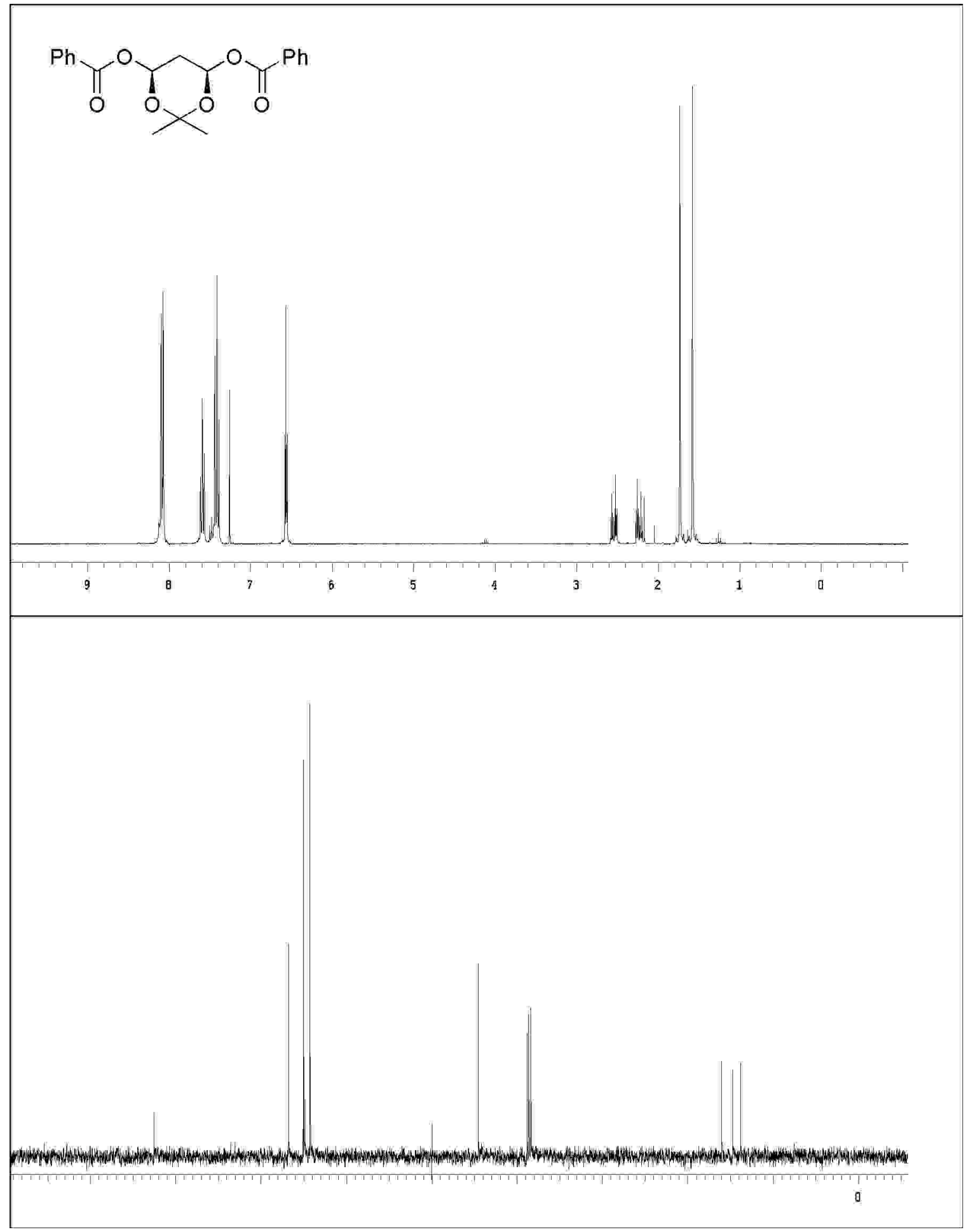
${ }^{1} \mathrm{H}$ NMR and ${ }^{13} \mathrm{C}$ NMR of 7
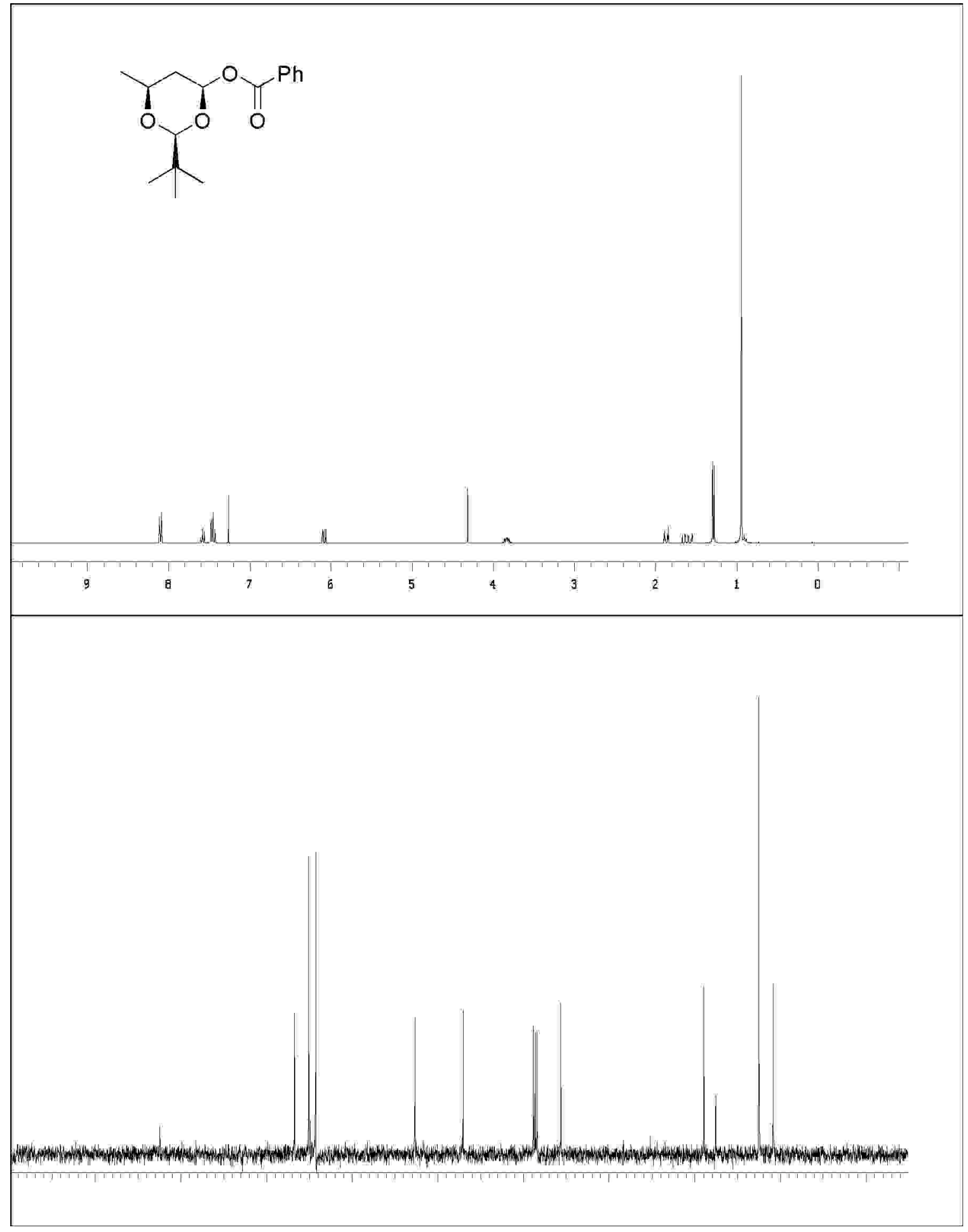


\section{${ }^{1} \mathrm{H}$ NMR and ${ }^{13} \mathrm{C}$ NMR of $\mathbf{1 1}$}
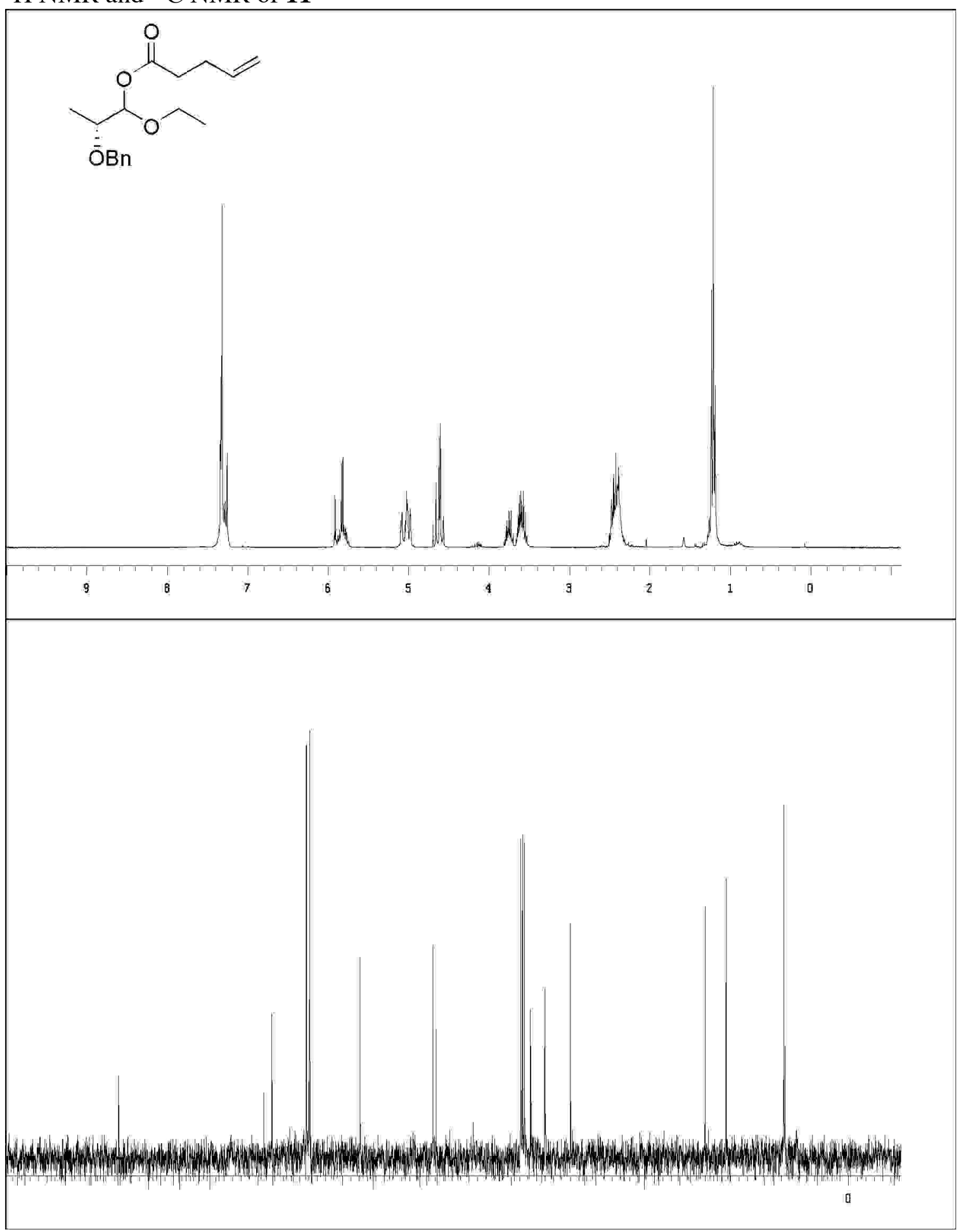
${ }^{1} \mathrm{H}$ NMR and ${ }^{13} \mathrm{C}$ NMR of $\mathbf{1 6}$
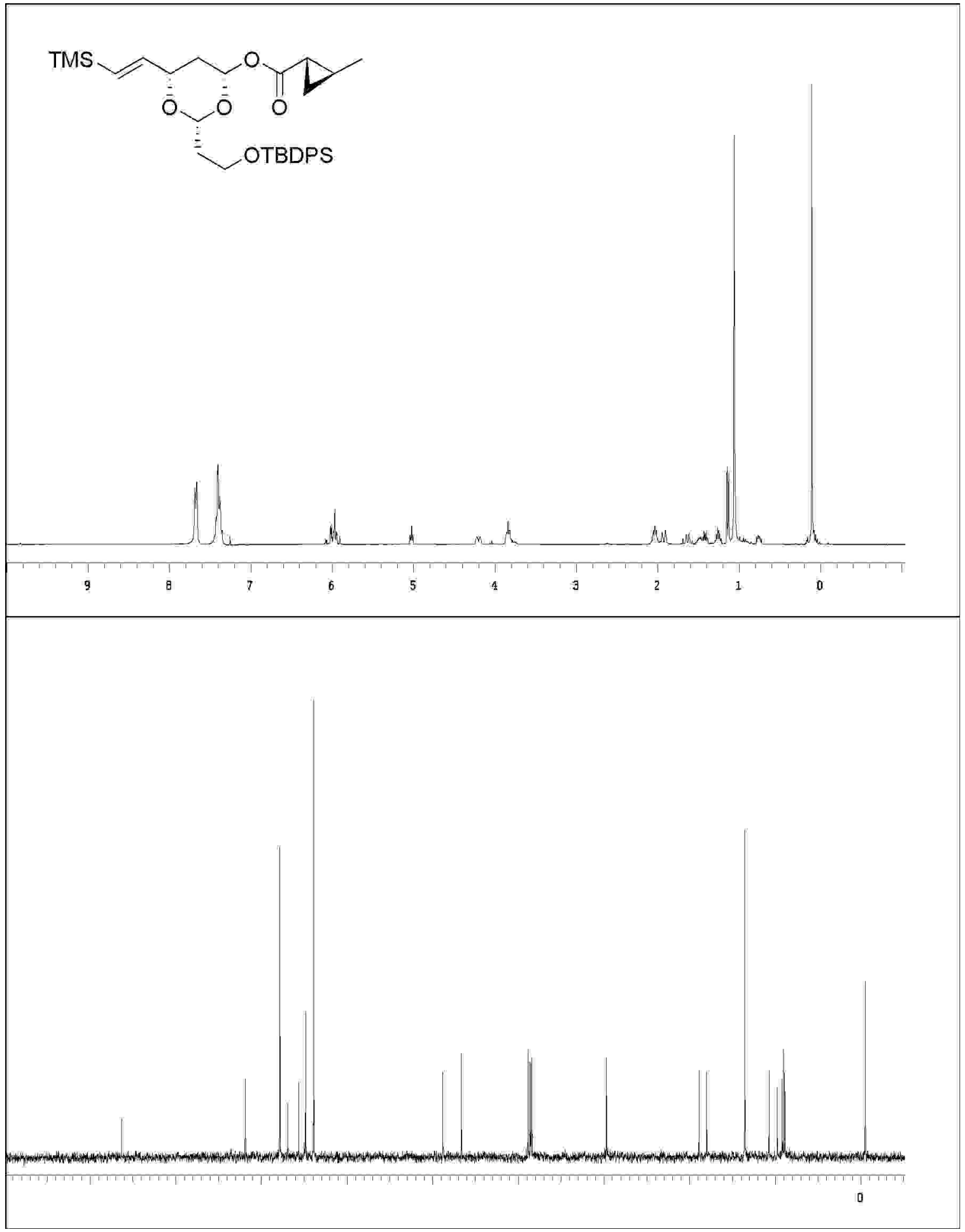